\title{
ARTÍCULOS
}

\section{EPIGRAFÍA DIGITAL: TECNOLOGÍA 3D DE BAJO COSTE PARA LA DIGITALIZACIÓN DE INSCRIPCIONES Y SU ACCESO DESDE ORDENADORES Y DISPOSITIVOS MÓVILES}

\author{
Manuel Ramírez-Sánchez, José-Pablo Suárez-Rivero y María-Ángeles \\ Castellano-Hernández
}

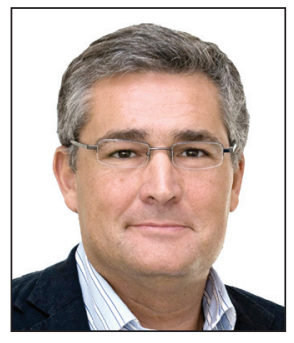

Manuel Ramírez-Sánchez es profesor titular de ciencias y técnicas historiográficas en la Facultad de Geografía e Historia de la Universidad de Las Palmas de Gran Canaria y director del Instituto Universitario de Análisis y Aplicaciones Textuales (latext). Sus líneas de investigación se centran en la historia de la cultura escrita en España y, en particular, en la epigrafía romana. http://orcid.org/0000-0002-4935-7313

Universidad de Las Palmas de Gran Canaria, Instituto Universitario de Análisis y Aplicaciones Textuales Campus Universitario del Obelisco, 35003 Las Palmas de Gran Canaria, España manuel.ramirez@ulpgc.es

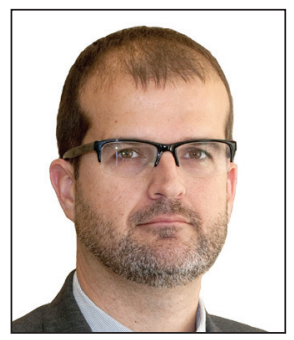

José-Pablo Suárez-Rivero, profesor titular de expresión gráfica en la ingeniería de la Escuela de Ingenierías Industriales y Civiles de la Universidad de Las Palmas de Gran Canaria. Director de la División de Matemáticas, Gráficos y Computación (MAGiC) y director de Política Informática de la universidad. Sus líneas de investigación se centran en las superficies basadas en mallas y geometría computacional, así como visualización 3D para dispositivos móviles y ordenadores. http://orcid.org/0000-0001-8140-9008

Universidad de Las Palmas de Gran Canaria, Instituto Universitario de Microlectrónica Aplicada Campus Universitario de Tafira, 35017 Las Palmas de Gran Canaria, España josepablo.suarez@ulpgc.es

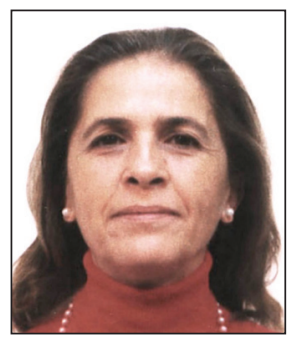

María-Ángeles Castellano-Hernández es conservadora jefe de la sección de arqueología romana del Museo Arqueológico Nacional, España. Ha comisariado varias exposiciones en Estados Unidos y Europa y colabora de manera directa con numerosas instituciones culturales. Sus líneas de investigación se centran en la epigrafía y en la escultura altoimperial. http://orcid.org/0000-0001-7454-0526

Museo Arqueológico Naciona Serrano, 13. 28001 Madrid, España angeles.castellano@mecd.es

\section{Resumen}

Se analizan las posibilidades del Image based modeling (IBM), como técnica de escaneado 3D de bajo coste para la modelización de inscripciones romanas, a partir del trabajo realizado en el Museo Arqueológico Nacional de Madrid sobre una amplia tipología de soportes epigráficos (piedra, bronce, arcilla), con resultados óptimos para la catalogación, estudio y difusión de este tipo de documentación histórica. Los resultados obtenidos permiten obtener inscripciones romanas en 3D que se pueden incorporar a los proyectos de epigrafía digital en curso, permitiendo su acceso a través de ordenadores y dispositivos móviles, sin coste añadido para los investigadores.

\section{Palabras clave}

Digitalización, Epigrafía, 3D, Fotografía digital, Inscripciones romanas, Bajo coste, Métodos, Tecnologías.

Title: Digital epigraphy: low-cost 3D technology provides online and mobile access to image-based modeling

\section{Abstract}

The possibilities of image-based modeling (IBM) as a low-cost 3D scanning technique for modeling Roman inscriptions are analysed. The study, carried out at the National Museum of Archaeology (Madrid), examined a wide range of Roman inscrip- 
tions (on stone, bronze and clay). The results obtained have proved to be excellent for cataloguing, studying and disseminating this type of historical documentation. The 3D Roman inscriptions can be incorporated into digital epigraphy projects in progress, allowing access via personal computers and mobile devices at no extra cost to researchers.

\section{Keywords}

Digitization, Epigraphy, 3D, Digital photography, Roman inscriptions, Personal computers, Mobile devices, Methods, Technologies.

Ramírez-Sánchez, Manuel; Suárez-Rivero, José-Pablo; Castellano-Hernández, María-Ángeles (2014). “Epigrafía digital: tecnología 3D de bajo coste para la digitalización de inscripciones y su acceso desde ordenadores y dispositivos móviles". El profesional de la información, septiembre-octubre, v. 23, n. 5, pp. 467-474.

http://dx.doi.org/10.3145/epi.2014.sep.03

\section{Introducción}

La epigrafía es, junto con la paleografía, una de las ciencias de la escritura que permite a los filólogos e historiadores leer documentos escritos desde la Antigüedad hasta nuestros días. La importancia de la documentación fotográfica en el oficio del epigrafista ha sido destacada en algunas obras básicas de la bibliografía especializada (Di-StefanoManzella, 1987), y todavía está muy extendida la costumbre de realizar calcos o vaciados para obtener reproducciones con la mayor fidelidad posible (Keppie, 1991).

Frente a la fotografía tradicional, bidimensional y limitada tan solo a aportar información de la altura y ancho de los soportes epigráficos, la representación tridimensional de las inscripciones permite mejorar la percepción de estos materiales, proporcionando acceso a la magnitud del grosor de los soportes, la decoración de las partes ocultas ante la cámara y otros aspectos relacionados con la estereometría de los epígrafes.

\section{La tecnología 3D de bajo coste puede impulsar la epigrafía digital}

Sin embargo, la tecnología de reproducción 3D aún no ha sido incorporada de manera efectiva en el trabajo de los epigrafistas debido a su alto coste y, sobre todo, a las limitaciones que imponen los propios soportes, que en muchas ocasiones no se conservan en los museos, sino que pueden estar dispersos en diversos lugares donde han sido reaprovechados (Buonopane et al., 2006).

Desde los años noventa del pasado siglo, la epigrafía ha llevado a cabo importantes proyectos de difusión a través de internet, sobre todo en el campo de las bases de datos online, entre las que destacan proyectos como la Epigraphische datenbank de la Universität Heidelberg, la Epigraphik-datenbank Clauss-Slaby, la base de datos Hispania epigraphica online, entre otros (Ramírez, 2003).

http://edh-www.adw.uni-heidelberg.de/home

http://www.manfredclauss.de

http://eda-bea.es

Pero el avance experimentado en la aplicación de la fotografía digital en la investigación epigráfica no ha sido tan importante, más allá del reemplazo de la fotografía tradicio- nal, como muestra la escasa calidad de las fotografías que aportan algunas de estas bases de datos.

Aunque se han propuesto varias técnicas para la reproducción 3D de calcos epigráficos (Barmpoutis et al., 2010; Bozia et al., 2012) o, más recientemente, algunos métodos de escaneado de inscripciones latinas a partir de imágenes múltiples (Lerma; Muir, 2014), aún no se ha desarrollado ninguno sobre los diversos soportes materiales de escritura, como son la piedra, la arcilla o el bronce. Por ejemplo, la utilización del láser escáner, que ha sido probada con éxito sobre algunos miliarios, no se ha aplicado en otros soportes debido a los problemas técnicos y a su elevado coste (Buonopane et al., 2006).

La utilización de una tecnología de bajo coste en la reproducción 3D de las inscripciones, como la que proponemos en este trabajo, puede impulsar estos proyectos de epigrafía digital, al incorporar en la investigación y difusión imágenes tridimensionales en cualquiera de los formatos estándar. Igualmente, estos modelos en 3D pueden ser utilizados en la formación de los futuros epigrafistas, como se ha propuesto para otros documentos patrimoniales (Pereira-Uzal; Robledano-Arillo, 2013), al permitir aproximar a los estudiantes a un repertorio de inscripciones que, de otra manera, sólo podrían ser estudiadas in situ.

\section{El proyecto Epigraphia 3D: divulgación de la colección epigráfica del Museo Arqueológico Nacional}

La antigua Roma produjo y publicó documentos legales, administrativos, comerciales, artísticos, familiares y privados, sobre distintos soportes materiales de escritura. Muchas de estas inscripciones realizadas en Hispania se conservan en el Museo Arqueológico Nacional de Madrid (en adelante $M A N)$. España posee un excepcional patrimonio epigráfico de época romana, integrado por varios millares de inscripciones que se conservan en museos dispersos por todo el país. El MAN, fundado en 1867 , se ha ocupado desde sus inicios de adquirir y conservar en sus fondos la colección de inscripciones romanas más importante de España, hecho al que contribuyó que durante muchos años fue el museo nacional de referencia.

Entre sus piezas destacan inscripciones procedentes de las principales ciudades romanas de la antigua Hispania, especialmente de la Bética y la Lusitania. La colección de 
bronces jurídicos es de las más importantes del mundo. En los últimos seis años el MAN ha experimentado una importante renovación museográfica y, después de llevar varios años cerrado al público, reabrió sus puertas en abril de 2014. En el nuevo montaje de las salas se ha dado una especial relevancia a las piezas arqueológicas que contienen epigrafía, al constituir la mejor fuente de información para conocer la vida romana en toda su extensión.

En el proyecto Epigraphia 3D se han escaneado 43 inscripciones romanas del MAN de diversos tipos, tales como

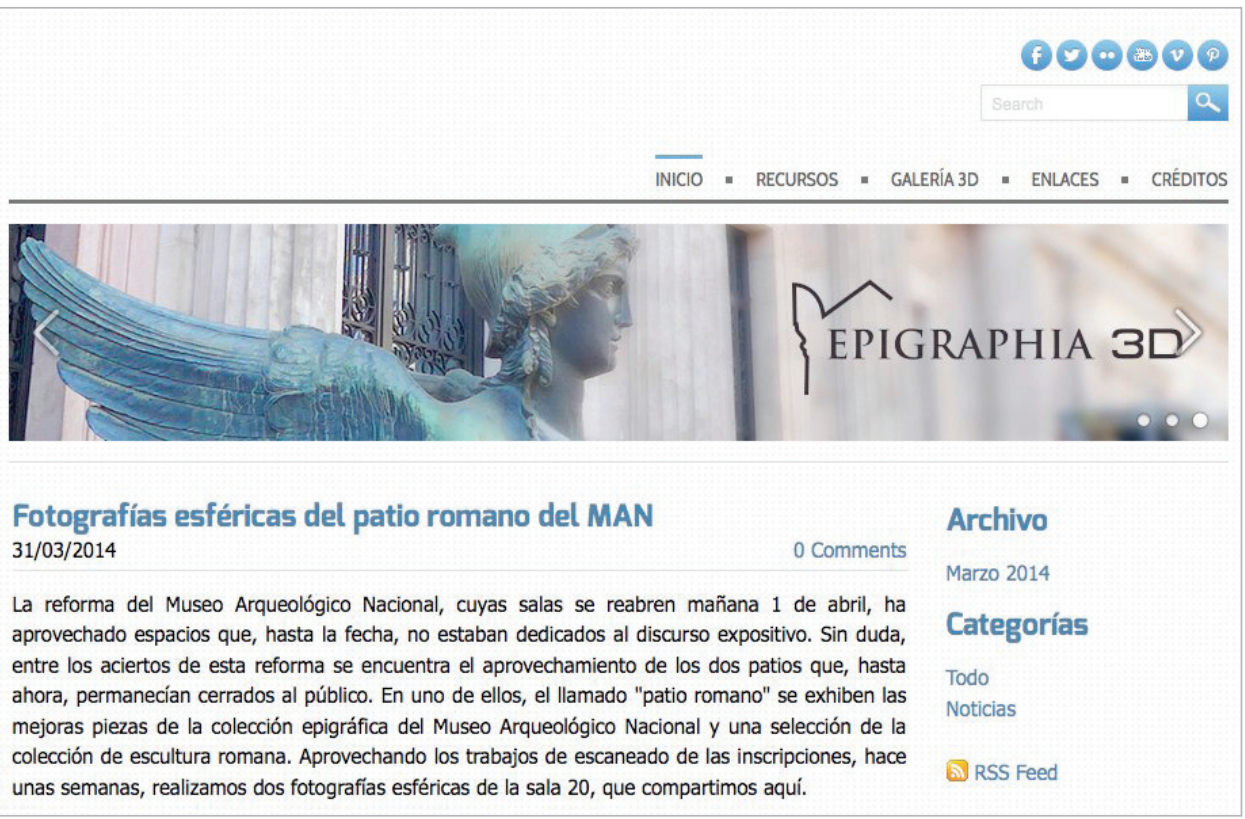
unas semanas, realizamos dos fotografías esféricas de la sala 20 , que compartimos aquí.

Figura 1. Página inicial de Epigraphia $3 D$

http://www.epigraphia3d.es funerarias, votivas, honorífi-

cas y monumentales. La mayoría de las inscripciones están realizadas en piedra, casi siempre mármol, aunque también se han modelado en $3 \mathrm{D}$ varios epígrafes realizados en piedra caliza o granito. Además, se han escaneado cuatro inscripciones realizadas en bronce (una tabula, una tessera hospitalis y dos sellos), dos inscripciones sobre ladrillos de arcilla y un mortero de arcilla con estampilla.

\section{El proyecto Epigraphia 3D ofrece más de cuarenta inscripciones romanas tridi- mensionales}

Es la primera que vez se afronta un proyecto de esta magnitud en el ámbito de la documentación epigráfica y los resultados abren una puerta a la accesibilidad de una técnica de bajo coste que puede ser incorporada a la investigación, y permite la difusión de este tipo de documentos históricos. El proyecto se divulga a través de una web en la que estos materiales están accesibles (figura 1).

http://www.epigraphia3d.es

\section{Metodología de escaneado 3D de las inscripciones mediante image based modeling (IBM)}

La visión tridimensional por ordenador incorpora un realismo sin precedente en la forma de visualizar e interactuar con ambientes gráficos, y acerca la realidad a lo digital y viceversa. En nuestro trabajo usamos un proceso para modelar objetos 3D desde fotografías, denominado image based modeling (IBM), que consiste en convertir una fotografía a 3D mediante el uso de técnicas fotogramétricas, realizando unas complejas operaciones matemáticas que permiten determinar la geometría tridimensional de los objetos. Esto se consigue mediante la combinación de imágenes individuales de diferentes puntos de vista, mediante un procedimiento que se conoce como pa- ralaje. En particular nuestra técnica IBM se basa en los principos de Structure from motion (SFM), que obtiene la estructura tridimensional de un objeto analizando la información o señales del movimiento a través de una variable temporal.

Esta técnica supone toda una revolución frente a la tradicional fotogrametría clásica que utiliza métodos matemáticos aún más complejos y un procesamiento de datos que, si bien procede de fotografías, lo hace bajo costosas tomas fotográficas referenciadas y con condiciones de difícil aplicación práctica.

La técnica de IBM permite generar un modelo basado en mallas que representa el objeto fotografiado. La malla es una superficie, normalmente de triángulos, que tiene fácil representación y visualización por ordenador.

Los principios generales de un sistema de este tipo son el aprovechamiento de las ubicaciones y correspondencias en las características de la imagen (puntos, líneas, esquinas, u otras características de alto nivel) en varias imágenes e inferir las localizaciones espaciales en 3D y las posiciones de la cámara.

Para los modelados que se realizan en el proyecto se ha utilizado Autodesk 123D Catch $^{\circ}$, un software libre que pone al alcance del usuario, de una forma sencilla, la posibilidad de realizar modelos 3D de cierta complejidad de modelado. http://www.123dapp.com/catch

Al ser el software libre y gratuito, no es necesario disponer de una licencia. Además, como el procesamiento se realiza en la nube, exime al usuario de contar con una máquina con gran capacidad de cálculo. Salvo que se manejen imágenes de gran tamaño, los datos a transferir no suponen horas de espera delante del ordenador.

Durante tres días de trabajo, en marzo de 2014, se han escaneado 43 inscripciones romanas del MAN preseleccionadas por su naturaleza, tipo y complejidad técnica para el trabajo, buena parte de las cuales estaban ya situadas en la exposición permanente. Las inscripciones de menor tamaño que estaban expuestas en vitrinas, así como algunas piezas que 


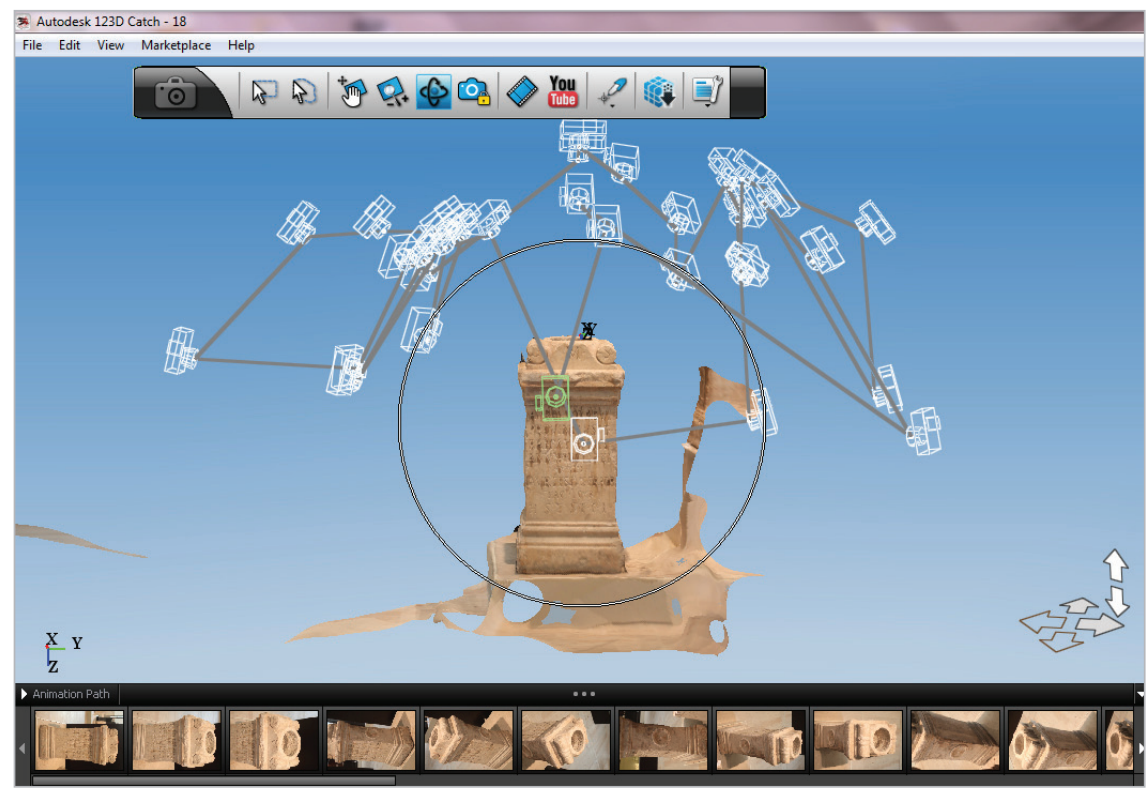

Figura 2. Ejemplo de inscripción fotografiada y el resultado generado con Autodesk $123 D$ Catch $^{\circ}$ tante cuidar que en las fotografías no aparezcan brillos o destellos producidos por la propia superficie del objeto. En el caso de las inscripciones romanas, el material no supone ninguna dificultad al tratarse de piedra o bronce, con superficies poco reflectantes.

\section{Procesamiento fotográfico con mo- delado 3D}

Utilizamos Autodesk 123D Catch ${ }^{\circ}$, que permite realizar modelos 3D de cierta complejidad de modelado de forma semiautomática mediante la combinación de fotografías convencionales. Finalizado el proceso se obtiene el objeto modelado en tres dimensiones, que se visualiza en la interfaz del programa. Además, se pueden observar las posiciones de la

se encontraban en los almacenes del Museo, fueron fotografiadas con la misma técnica que el resto de las inscripciones. Se han utilizado diferentes tipos de luz para comprobar el grado de importancia de este factor en el trabajo de campo. Los mejores resultados se han obtenido con luz natural en la sala 20 (patio romano) del Museo. Los resultados obtenidos con focos halógenos han sido menos satisfactorios.

\subsection{Flujo de trabajo, procesamiento de fotografías y reconstrucción 3D}

A continuación se describe el flujo de trabajo de forma que pueda ser replicado por cualquier investigador familiarizado con conceptos muy básicos de informática y fotografía a nivel de usuario:

\section{Toma de fotografías del objeto}

Este primer paso en sí mismo no encierra grandes complicaciones. Únicamente se precisa que el objeto esté situado de forma que se pueda fotografiar a su alrededor y en todos los ángulos. Asimismo es importante contar con buena luz neutra (blanca) sin que incida directamente sobre el objeto, para evitar que genere sombras, reflexiones, etc. Es imporgrafías dependiendo de la complejidad del objeto, que con- toma de las fotografías y las imágenes utilizadas.

Suele ser suficiente con una muestra de entre 12 y 40 fototemplen la figura desde varios ángulos, incluidas las vistas "desde arriba", y "desde abajo", si procede, para poder completar el objeto en tres dimensiones. El promedio fue de 32 fotografías por inscripción. Al final del proceso obtenemos un fichero con extensión nativa Autodesk.3dp (figura 2).

\section{Edición y reparación de la malla del objeto}

Al finalizar el paso anterior se habrá obtenido una malla o superficie basada en triángulos que representa la geometría tridimensional de la inscripción. La formación de mallas de triángulos ha demostrado ser en este campo y en muchos otros de la ciencia y la ingeniería la mejor estructura para representar digitalmente objetos en 3D. Cuando se obtiene el objeto modelado en Autodesk 123 D Catch $^{\circledR}$ es necesario comprobar minuciosamente la malla por si existieran imperfecciones que sea necesario modificar. Además, el objeto siempre tiene un punto de apoyo que no se puede fotografiar y se ha de corregir y añadir una base reconstruida. Para ello hemos utilizado Blender, un software de código abierto (figura 3). http://www.blender.org

Una inspección minuciosa a la malla obtenida permite concluir que se trata de una superficie adecuada en relación al número de puntos y triángulos y las singularidades geométricas del objeto, lo que suele denominarse como adaptatividad de la malla (Suárez et al., 2001). En las zonas con poca curvatura la malla tiene pocos puntos, mientras que en otros zonas de la inscripción

Figura 3. Triangulación que conforma el objeto Blender ${ }^{\circ}$ 

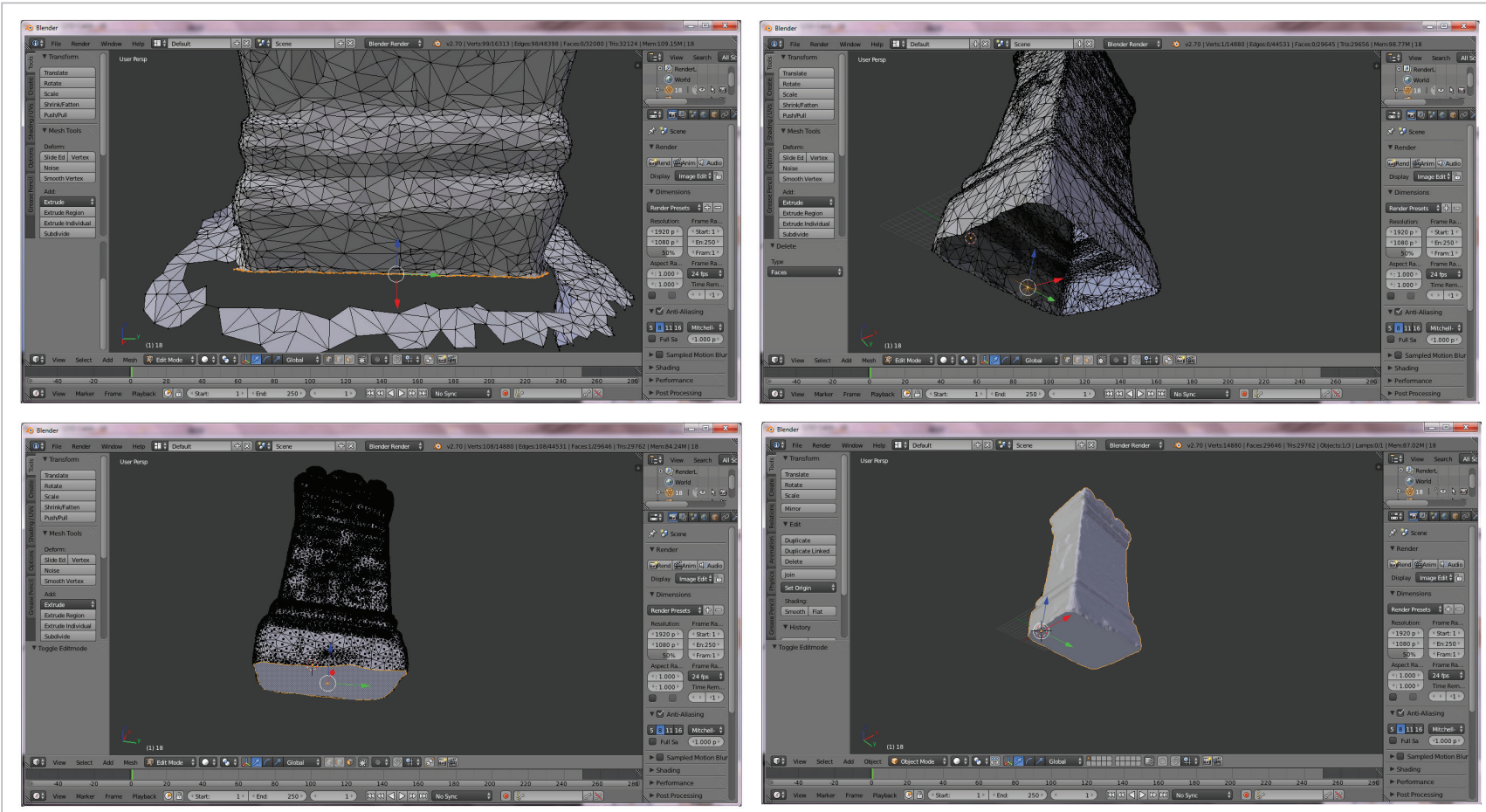

Figura 4. Distintas perspectivas y operación de recorte de la base de la inscripción

se necesita mayor definición y por tanto se utilizan más puntos y triángulos.

Una de las tareas más problemáticas es el recorte de la malla que afecta a la parte sobrante del suelo o soporte de la inscripción, que realiza mediante la selección cuidadosa de triángulos y puntos que no forman parte de la inscripción. En su lugar se recubre la inscripción ya cortada con un plano simulado de forma que cierre la geometría topológica del objeto (figura 4).

\section{Tratamiento de texturas con un editor de gráficos}

De la fase anterior se obtiene un archivo en formato jpg con las texturas que definen, entre otras cosas, el perímetro de la base que se le ha añadido al objeto. Mediante un editor de gráficos se clona la textura, de tal manera que la parte reconstruida se asemeje lo más posible a la realidad. En nuestro proyecto hemos utilizado Photoshop CS4 $4^{\circ}$, pero se puede utilizar cualquier software alternativo. En esta etapa podemos mejorar la calidad de la textura, que incidirá positivamente en el realismo del modelado.

\section{Visualización final y publicación en la nube}

El último paso del proceso es la publicación del objeto. Desde 2012 existen opciones para publicar estos formatos en la nube, como p3d, Sketchfab o Verold Studio. http://p3d.in https://sketchfab.com http://verold.com

Estas plataformas ofrecen alojamiento gratuito y posibilidades para compartir los modelos 3D, constituyendo una alternativa económica para investigadores e instituciones. En nuestro proyecto hemos utilizado Sketchfab. https://sketchfab.com/epigraphia3d

La visualización 3D se ha desarrollado en los últimos años gracias a la tecnología WebGL, un estándar que permite visualizar gráficos en 3D en navegadores web multiplataforma. WebGL es promovido por un consorcio de tecnología sin ánimo de lucro denominado Khronos Group y permite mostrar gráficos en 3D acelerados por hardware (GPU) sin la necesidad de plug-ins, con el único requisito de que el navegador soporte OpenGL 2.0 u OpenGL ES 2.0. Técnicamente se trata de una API accesible mediante Javascript que permite usar la implementación nativa de OpenGL ES 2.0.

SketchFab se encarga de ocultar y hacer trasparente al usua-

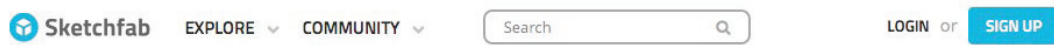

18

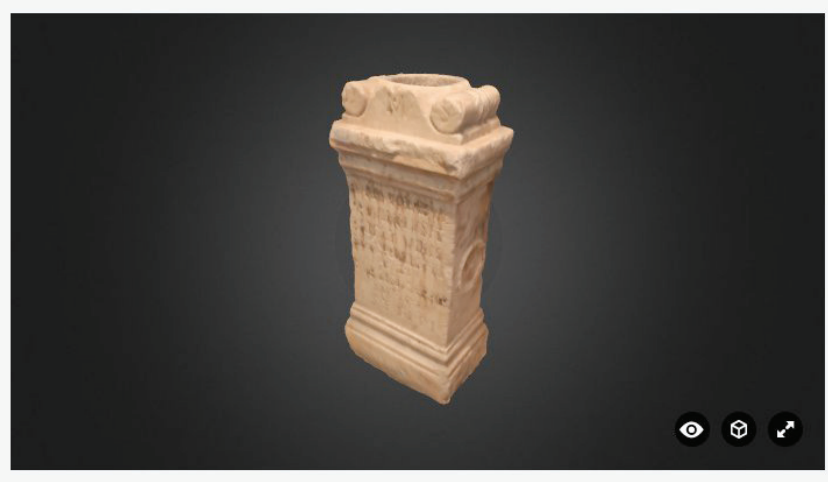

$\star 0 \odot 2$

EMBED

$\rightarrow$ SHARE + FAYORITE

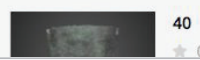

Figura 5. Visualización mediante Sketchfab de una inscripción en 3D (navegador Firefox) 


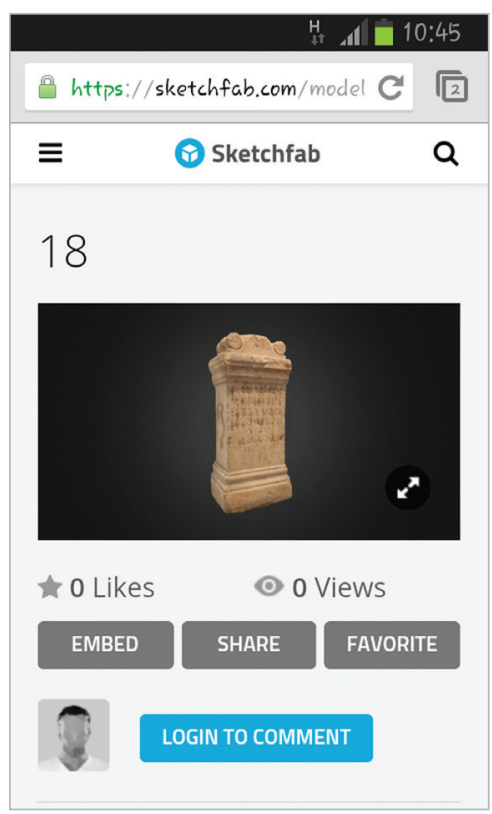

Figura 6. Visualización mediante Sketchfab de inscripción en dispositivo móvil Android (Samsung S3)

rio la codificación de la malla del objeto al código WebGL que lo muestra en una pantalla del navegador, tanto en ordenadores personales como en dispositivos móviles (figuras 5 y 6 ).

\section{Representación digital de las inscripciones 3D: formatos de archivo}

Existe una gran variedad de formatos de archivo que permiten representar objetos tridimensionales, pero no todos son abiertos o libres, o han sobrevivido a los constantes cambios y avances en software y los métodos asociados. OBJ es un formato de archivo de definición de geometría elaborado inicialmente por Wavefront Technologies. Es abierto y ha sido adoptado por otros proveedores de aplicaciones de gráficos 3D, por lo que está aceptado universalmente.

OBJ representa sólo la geometría 3D: la coordenada espacial de cada vértice, las coordenadas $u$ y $v$ de los vértices de textura, las "normales" de cada vértices y las caras o polígonos (triángulos en nuestro caso) definidos como una lista de vértices, y vértices textura. Las letras $u$ y $v$ indican los ejes de la textura 2D porque $x$, y y $z$ ya se utilizan para los ejes del objeto original 3D.

Los vértices se almacenan en sentido contrario a las agujas del

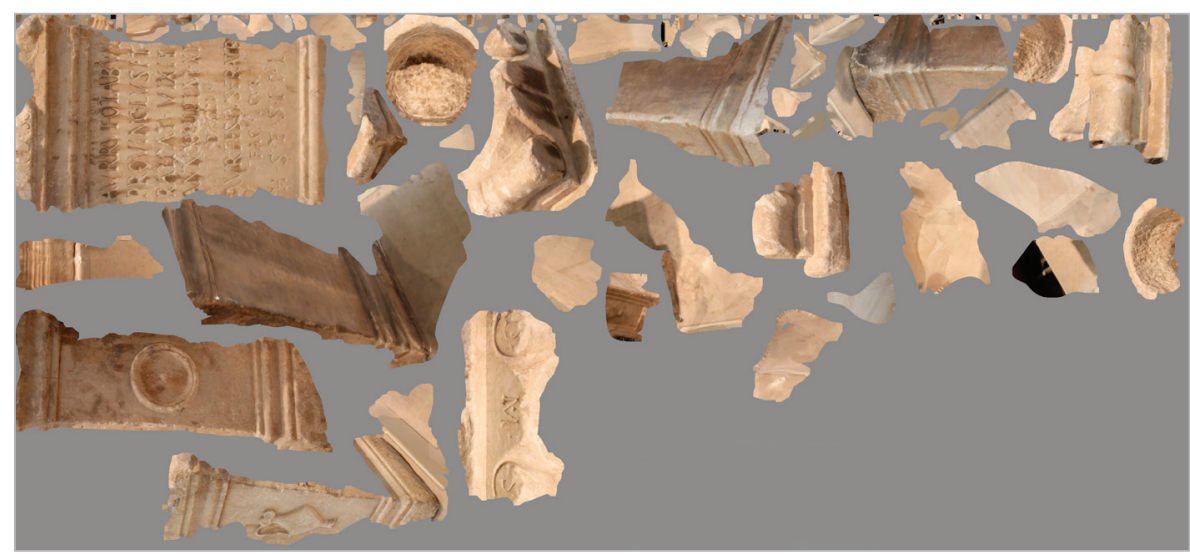

Figura 7. Archivo jpg con la forma organizada todas las texturas y extractos de las fotografías iniciales procesadas reloj de forma predeterminada, por lo que la declaración explícita de caras normales es innecesaria. Las coordenadas OBJ no tienen unidades, pero los archivos OBJ pueden contener información de escala en una línea de comentario. A continuación se muestra un esquema de la estructura del formato $O B J$ :

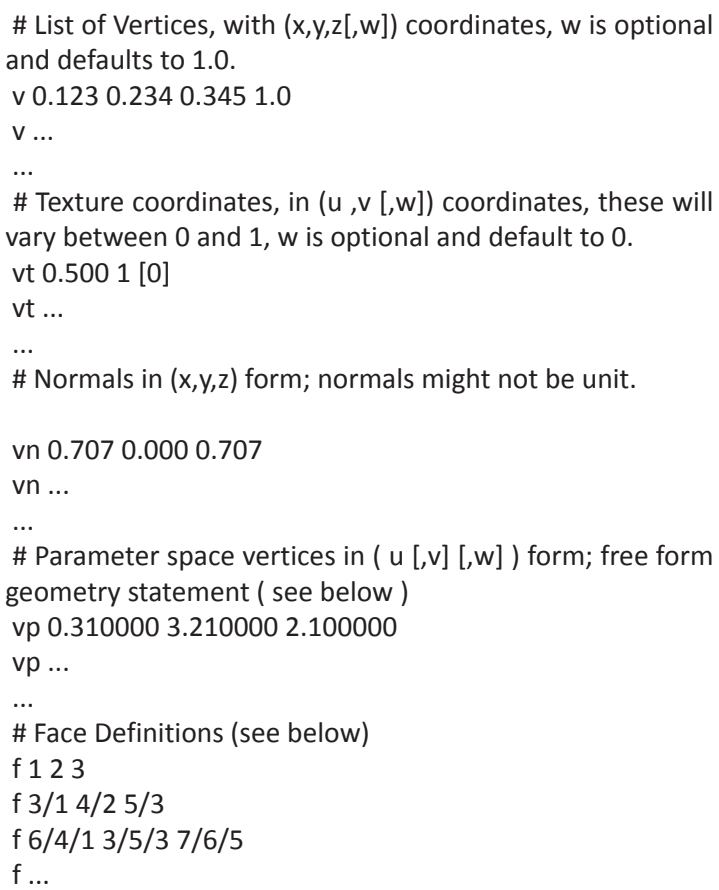

En los gráficos 3D por ordenador, otra parte bien diferenciada e igual de importante que la parte geométrica es la que compone las características de los materiales de la superficie que se representa. El formato del archivo MTL (material template library) es un formato de archivo complementario que describe el sombreado de la superficie del material, y las propiedades de los objetos dentro de uno o más archivos OBJ. Un archivo OBJ puede hacer referencia a uno o más archivos MTL, llamados "bibliotecas de materiales". Esta parte de la representación es fundamental para darle el realismo adecuado a la escena $u$ objeto que se representa.

El formato MTL es un estándar definido también por Wavefront Technologies, y se trata de ficheros ascii con las propiedades de reflexión de la luz de una superficie a los efectos de representación en la pantalla del ordenador, de acuerdo con el modelo de reflexión de Phong ${ }^{1}$. En este formato se especifica el archivo imagen raster que define la textura de la inscripción, que debe encontrarse en disco a la par que el fichero OBJ y el propio MTL. Suele ser un archivo jpg, que recoge de forma organizada todas las texturas, o extractos de las fotografías iniciales procesadas, que se almacenan en este fichero, como se puede ver en la figura 7 .

Al interpretar el ordenador tanto el archivo OBJ como el MTL, se realiza la carga y mapeo de la textura a la geometría de la inscripción. Se trata en realidad de 
una norma que tiene un amplio apoyo entre los diferentes fabricantes de software por lo que es un formato útil para el intercambio de materiales. Para tener una idea de la estructura de este formato, se ilustra a continuación mediante un ejemplo:

newmtl Texture_0 \# define a material named Texture_0
Ka 1.0000001 .0000001 .000000 \# white
Kd 1.0000001 .0000001 .000000 \# white
Ks 0.0000000 .0000000 .000000 \# black (off)
d 1
Ns 0.000000
illum 1
map_Kd 2_tex_0.jpg \# the diffuse texture map
newmtl Untextured
Ka 0.5019610 .5019610 .501961
Kd 0.5019610 .5019610 .501961
Ks 0.0000000 .0000000 .000000
d 1
Ns 0.000000
illum 1

\section{Comprobación del grado de precisión de los modelos 3D}

Una de las ventajas del image based modelling (IBM) es que es muy simple, económico y rápido. Pero además demostramos que es preciso en relación a las mallas superficiales obtenidas, ya que los modelos 3D resultantes representan con fidelidad las proporciones de las inscripciones originales, como hemos podido comprobar a partir del siguiente experimento:

1. Hemos realizado varias mediciones en una selección de inscripciones, tomando distancias en $x$ e $y$.

2. Posteriormente hemos realizado las mismas medidas en $x$ e $y$ sobre los correspondientes modelos 3D, usando como base de visualización y medida Autodesk 123D Catch $^{\circledast}$ y Blender ${ }^{\circledast}$.

3. Finalmente, hemos calculado los cocientes entre las diferentes medidas, obteniendo un ratio o proporción, adimensional, que nos ha permitido comparar la precisión entre las medidas reales y las obtenidas usando los modelos 3D.

Al objeto de sintetizar, mostramos en la figura 8 una de las inscripciones romanas escogidas para realizar los cálculos, con indicación de los elementos medidos. Es importante destacar la elección combinada de distancias en los dos ejes direccionales horizontal y vertical, que es fundamental para obtener la información geométrica en modo de proporciones básicas $x$ e $y$ de la inscripción.

La tabla 1 muestra las medidas tanto originales obtenidas sobre la inscripción original, así como las tomadas en Blen$\operatorname{der}^{\circledR}$, usando medida propia de pantalla, y las obtenidas $A$ utodesk 123D Catch $^{\circledR}$. A pesar de que las unidades métricas no son las mismas, debido a la escala por defecto que adopta el software empleado, son útiles para calcular la proporción según los tres tipos de medidas.

En la tabla 2 se muestran los cocientes obtenidos para cada medida $x, y, p, f, a$. Se observa que por columna, los cálculos del cociente se encuentran dentro de una exactitud hasta la primera o tercera cifra decimal. La similitud de estas magnitudes, en comparación, nos indican que las medidas son similares y se encuentran dentro de una tolerancia admisible. Aunque no calculamos tolerancias específicas de las medidas, hemos asegurado que los cálculos de la proporción están en consonancia con la definición visual obtenida en el ordenador. Dicho de otra forma, al comparar las medidas reales tomadas con escalímetro y las medidas obtenidas sobre el modelo mediante Autodesk 123D Catch $^{\circledR}$ y Blender ${ }^{\circledast}$, comprobamos que no hay diferencias sustanciales en el modelo tomado digitalmente. Asimismo, adicionalmente a la similitud en medidas, nos está indicando preservación de la proporción, lo cual es relevante para concluir que las proporciones se preservan durante el proceso de modelado.
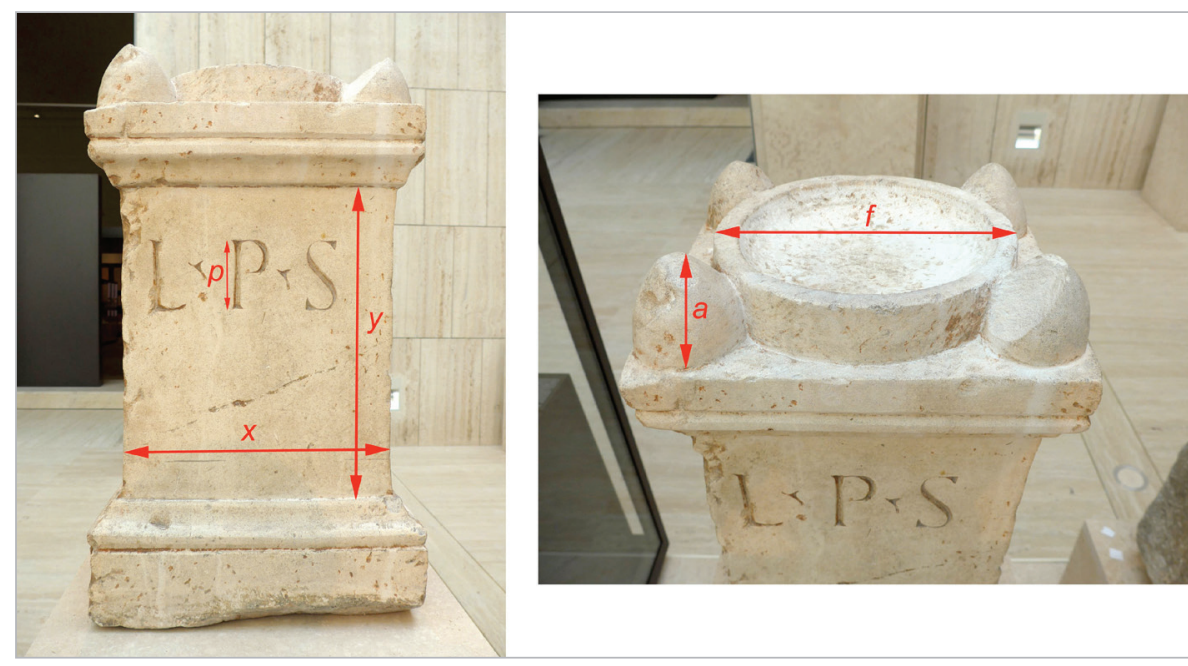

Figura 8. Medidas obtenidas del ara votiva consagrada a Liber Pater, Museo Arqueológico Nacional (no inventario: 16518)

Tabla 1. Medidas obtenidas de la inscripción de la figura 8. La fila 1 representa la medida original tomada con escalímetro en $\mathrm{cm}$, la fila 2 la medida del modelo en $B l e n d e r^{\circledR}$, usando medida propia de pantalla en Blender, y la fila 3 la medida con Autodesk $123 D$ Catch $^{\circledR}$ en mm

\begin{tabular}{|l|c|c|c|c|c|}
\hline & Altura $(\boldsymbol{y})$ & Ancho $(\boldsymbol{x})$ & $\begin{array}{c}\text { Altura letra } \\
\mathbf{P}(\boldsymbol{p})\end{array}$ & $\begin{array}{c}\text { Ancho } \\
\text { focus }(\boldsymbol{f})\end{array}$ & $\begin{array}{c}\text { Alto panecillo } \\
(\boldsymbol{a})\end{array}$ \\
\hline Original $(\mathrm{cm})$ & 29,7 & 26 & 6,5 & 23 & 6,5 \\
\hline Blender $(\mathrm{udB})$ & 20,553 & 17,953 & 4,709 & 15,647 & 4,072 \\
\hline Autodesk Catch $(\mathrm{mm})$ & 20,42 & 17,95 & 4,36 & 15,7 & 4,05 \\
\hline
\end{tabular}

Tabla 2. Cocientes obtenidos a partir de las distancias recogidas en la tabla 1

\begin{tabular}{|l|c|c|c|c|c|c|}
\hline & $\boldsymbol{x} / \boldsymbol{y}$ & $\boldsymbol{p} / \boldsymbol{y}$ & $\boldsymbol{p} / \boldsymbol{x}$ & $\boldsymbol{a} / \boldsymbol{f}$ & $\boldsymbol{f} / \boldsymbol{x}$ & $\boldsymbol{f} / \boldsymbol{y}$ \\
\hline Original $(\mathrm{cm})$ & 0,875 & 0,218 & 0,250 & 0,282 & 0,884 & 0,774 \\
\hline Blender $(\mathrm{udB})$ & 0,873 & 0,229 & 0,262 & 0,260 & 0,871 & 0,761 \\
\hline Autodesk Catch $(\mathrm{mm})$ & 0,879 & 0,213 & 0,242 & 0,257 & 0,874 & 0,768 \\
\hline
\end{tabular}


Por motivos de extensión no incluimos en este artículo los datos concernientes a las otras cinco inscripciones sometidas a este estudio numérico, de las cuales hemos obtenido las mismas conclusiones presentadas en este texto.

\section{Conclusiones}

El uso de la tecnología 3D en la difusión del patrimonio epigráfico es una realidad accesible al investigador mediante tecnologías de bajo coste como la que se ha aplicado en el proyecto Epigraphia 3D. Durante buena parte del siglo XIX los epigrafistas recurrieron a los calcos y a los vaciados en yeso para reproducir las inscripciones, mientras que a finales de la centuria y, sobre todo, a partir del siglo XX la fotografía se convirtió en uno de los elementos indispensables de la investigación epigráfica. Ha sido en las últimas décadas cuando, a raíz de la generalización de las cámaras digitales, muchos epigrafistas han alcanzado su independencia con respecto a los fotógrafos profesionales que, hasta entonces, eran su mejor aliado en el trabajo de campo. La utilización de técnicas de digitalización y modelado $3 \mathrm{D}$ como la que hemos mostrado en este trabajo permitirá dar un paso más a los investigadores, permitiéndoles acceder a mejores medios para reproducir las inscripciones.

Los proyectos en curso sobre epigrafía digital y las bases de datos epigráficas en línea, descritas al comienzo de este artículo, pueden incorporar estos formatos abiertos en 3D para facilitar a los investigadores y estudiosos un acceso más fiable que a través de las series de fotografías que, en muchas ocasiones, carecen de la calidad necesaria para su estudio. En suma, se trata de una tecnología que puede revolucionar en las próximas décadas un campo de estudio de larga tradición en las humanidades.

Algunas revistas científicas de arqueología, como el Journal of archaelogical science, acaban de incorporar en su web visores 3D que permiten publicar los formatos Wavefront file (OBJ), Stanford triangle format (PLY) o Universal 3D file (U3D), para facilitar a los autores un medio más para difundir los resultados de sus investigaciones.

http://www.journals.elsevier.com/journal-of-archaeological-science

Aunque es posible que haya que esperar aún varios años para ver generalizada esta iniciativa en las mejores revistas científicas de artes y humanidades, es indudable que para los epigrafistas será un elemento indispensable en su formación el manejo de formatos estandarizados de modelos 3D.

A partir del estudio numérico de distancias concretas tomadas en las inscripciones, así como en las mismas distancias tomadas en el software empleado (Autodesk 123D Catch $^{\circledR}$ y Blender $\left.^{\circledR}\right)$, hemos podido comprobar que nuestros modelos guardan precisión y proporción respecto de las inscripciones originales. Como trabajo futuro, para desarrollar un minucioso estudio de la calidad de los modelos epigráficos obtenidos, nos proponemos el estudio de las escalas obtenidas en la digitalización y además, el estudio de la fiabilidad del color mediante técnicas de comparación y gestión del color.

\section{Nota}

1. El modelo de reflexión de Phong fue inventado por Bui-Tuong Phong en la Universidad de Utah en 1973. Lo publicó en conjunción con un método conocido como sombreado Phong, muy usado en muchas aplicaciones por su eficiencia en tiempo de proceso.

\section{Agradecimientos}

1) Agradecemos el trabajo de recogida de datos realizado por Haridian Guerra-Soto y Alfredo Hernández-Febles. Agradecemos también al Museo Canario (Las Palmas de Gran Canaria) habernos permitido realizar en sus instalaciones varias simulaciones previas de la técnica de trabajo antes de su utilización en el Museo Arqueológico Nacional en Madrid.

2) Trabajo financiado con cargo al proyecto FCT-13-6025 "Descifrando inscripciones romanas en 3D: ciencia epigráfica virtual", financiado por la Fundación Española para la Ciencia y la Tecnología - Ministerio de Economía y Competitividad, y la Universidad de Las Palmas de Gran Canaria.

\section{Bibliografía}

Barmpoutis, Angelos; Bozia, Eleni; Wagman, Robert S. (2010). "A novel framework for 3D reconstruction and analysis of ancient inscriptions". Journal of machine vision and applications, v. 21, n. 6, pp. 989-998.

http://www.digitalworlds.ufl.edu/angelos/file.php?f=barmpoutis_ mva10.pdf

http://dx.doi.org/10.1007/s00138-009-0198-7

Bozia, Eleni; Barmpoutis, Angelos; Wagman, Robert S. (2012). "The first online 3D epigraphic library of Florida digital epigraphy and archaeology project". En Procs of $14^{\text {th }}$ Int congress of Greek and Latin epigraphy. Berlin: Humboldt University - Aiegl

Buonopane, Alfredo; Grossi, Piergiovanna; Guarnieri, Alberto; Pirotti, Francesco (2006). "L'impiego del laser scanner nel rilievo delle iscrizioni sui miliari". En BertinelliAngeli, Maria-Gabriella; Donati, Angela. Misurare il tempo. Misurare lo spazio. Atti del Colloquio Aiegl-Borghesi 2005. Faenza: Fratelli Lega Editori, pp. 373-388. ISBN: 8875940940

Di-Stefano-Manzella, Ivan (1987). Mestiere di epigrafista. Guida alla schedatura del materiale epigrafico lapideo. Roma: Edizioni Quasar

Keppie, Lawrence (1991). Understanding roman inscriptions. Baltimore: Johns Hopkins University Press. ISBN: 0801843529

Lerma, José-Luis; Muir, Colin (2014). “Evaluating the 3D documentation of an early Christian upright stone with carvings from Scotland with multiples images". Journal of archaeological science, v. 46, pp. 311-318. http://dx.doi.org/10.1016/j.jas.2014.02.026

Pereira-Uzal, José-Manuel; Robledano-Arillo, Jesús (2013). "Uso de tecnologías 3D en la digitalización y difusión de documentos de alto valor patrimonial". El profesional de la información, v. 22, n. 3, pp. 215-223. http://dx.doi.org/10.3145/epi.2013.may.04

Ramírez-Sánchez, Manuel (2003). "Recursos de epigrafía y numismática en internet: balance actual y perspectivas en España". Boletín Millares Carlo, v. 22, pp. 275-301.

Suárez-Rivero, José-Pablo; Carey, Graham; Plaza, Ángel (2001). "Graph based data structures for skeleton based refinement algorithms". Communication in numerical methods in engineering, v. 17, n. 12, pp. 903-910.

http://dx.doi.org/10.1002/cnm.460 